\title{
Combined malonic and methylmalonic acidemia
}

INSERM

\section{Source}

INSERM. (1999). Orphanet: an online rare disease and orphan drug data base. Combined malonic and methylmalonic acidemia. ORPHA:289504

Combined malonic and methylmalonic acidemia is a rare inborn error of metabolism characterized by elevation of malonic acid (MA) and methylmalonic acid (MMA) in body fluids, with higher levels of MMA than MA. CMAMMA presents in childhood with metabolic acidosis, developmental delay, dystonia and failure to thrive or in adulthood with seizures, memory loss and cognitive decline. 